\title{
Effect of reduced photon count levels and choice of normal data on semi-automated image assessment in cardiac SPECT: Doing more with fewer counts
}

\author{
Marina Piccinelli, PhD, ${ }^{a}$ and James Galt, $\mathrm{PhD}^{\mathrm{a}}$ \\ a Department of Radiology and Imaging Sciences, Emory University School of Medicine, Atlanta, \\ GA
}

Received Oct 15, 2018; accepted Oct 16, 2018

doi: $10.1007 / \mathrm{s} 12350-018-01499-6$

See related article, pp. 1469-1482

The use of imaging in cardiology has been growing steadily in the last decades. While new noninvasive methodologies have been gaining importance in clinical practice-such as computed tomography or cardiac magnetic resonance-nuclear myocardial perfusion imaging (MPI) and particularly single-photon emission computed tomography (SPECT) remain the most established technique for cardiac stress testing and assessment of a variety of cardiovascular diseases.

A major issue connected with the use of nuclear MPI is the radiation dose associated with these diagnostic tests. Even if the risk-benefit ratio of selecting a nuclear imaging procedure is favorable ${ }^{1}$ if compared with the consequences of a misdiagnosis, there is a widespread perception in the medical community as well as in the general public that nuclear imaging is associated with overly high radiation doses. Currently, a standard rest-stress ${ }^{99 \mathrm{~m}}$ Tc SPECT study is performed with a cumulative radiation dose of $\sim 12 \mathrm{mSv}$ (compared to the average natural background radiation of about $3 \mathrm{mSv}$ ), although large differences worldwide have been recently documented ${ }^{2}$ by the International Atomic Energy Agency (IAEA) in terms of safety practices and effective radiation dose.

Reprint requests: James Galt, PhD, Department of Radiology and Imaging Sciences, Emory University School of Medicine, Atlanta, GA; jgalt@emory.edu

J Nucl Cardiol 2020;27:1483-5.

$1071-3581 / \$ 34.00$

Copyright (c) 2018 American Society of Nuclear Cardiology.
The nuclear cardiology community (SNMMI, ASNC) has forcefully responded to these concerns by promoting guidelines that encourage laboratories to greatly decrease radiation burden and developing new technologies and methods to minimize injected doses and acquisition times. ${ }^{3}$ The introduction of stress-only or stress-first protocols can significantly decrease the cumulative injected dose per study. New algorithms for image reconstruction with resolution recovery and noise reduction provide images with higher contrast and improved quality, effectively enabling half-dose or quarter-dose protocols. High-efficiency cameras with solid state detectors and novel collimators show a several-fold increase in count sensitivity which can in turn allow reduction in dose and imaging time.

All these advancements are certainly welcome to unsure patients' safety and crucial for the continued success of the nuclear cardiology field, but caution should be exercised in the routine clinical application of low- or ultra-low-dose imaging protocols. First and foremost, MPI diagnostic power should not be compromised by dose reduction. Secondly, automated quantification of MPI studies, an essential component of perfusion assessment, is based on the comparison of the patient's scan to a normal database. ${ }^{4}$ As low-dose imaging protocols obtained with new scanners steadily enter clinical practice, so the definition of appropriate normal limits becomes of paramount importance. Yet, investigations on the effect of dose reduction and normal database selection compared to standard MPI protocols, particularly in the same patient population, remain limited. ${ }^{5-7}$

In the present issue of Journal of Nuclear Cardiology, Wetzl et al. ${ }^{8}$ addressed the crucial issue of normal database selection in the context of low-dose imaging protocols. In fact, in addition to the well-known 
parameters affecting the normal database definition and creation such as tracer, type of camera, clinical protocol, and gender, the count level may be a further source of inconsistency between patient's data and normal reference and ultimately introduce bias into perfusion quantification. From standard full-count acquisitions, the authors artificially created progressively lower-count datasets by subsampling the original projection data for both patients and normal datasets. To assess the effect of normal database definition on the number of hypoperfused/normal segments reclassification and ultimately diagnostic performance and feasibility of low-dose protocols, image quantification was performed for all reduced-dose images with both count-matched and fullcount normal databases. When full-count normal database was used, higher summed stress scores (SSS) and a trend in patient- and segment-based reclassification towards hypoperfusion and high risk was evidenced. Conversely, with matched-count database, a progressive decrease in SSS and reclassifications towards normal perfusion was described. When the clinical significance of increased false positive rate (full-count database) or false negative rate (matched-count database) was considered, the authors claimed that a reduction of up to $67 \%$ of the standard injected doses could be safely implemented.

Nevertheless, with major reductions in radiation doses (i.e., below 8.3 counts per pixel), all reclassifications became markedly significantly different with respect to diagnostic results on full-count datasets, emphasizing the need for care in the selection of reduced-dose imaging protocols. Their results confirm previous publications assessing the introduction of total perfusion defect (TPD) bias as a function of decreasing count levels. ${ }^{4,6}$ In Nakazato et al., ${ }^{6}$ authors quantified TPD with count-specific vs standard normal limits with lowering injected doses for a high-sensitivity cadmiumzinc-telluride (CZT) dedicated cardiac SPECT system (D-SPECT; Spectrum Dynamics). Lowering dose protocols are indeed associated with increased TPD bias for both count-specific and standard normal limits, although the use of matched normal databases seems to decrease its impact on image quantification and MPI diagnostic power. It may be worth mentioning that Nakazato et al. ${ }^{6}$ created the lower-dose datasets by reframing list-mode data, a method that may more accurately model a lowcount study than simulated low-count data.

These studies examine high-sensitivity systems with unique collimation and utilize SPECT reconstruction software specifically designed for that system. The effects of a significant reduction in counts on other systems must be investigated independently. Also, one must not extrapolate these results to the use of SPECT reconstruction software designed to allow reconstruction with lower counts through the use of resolution modeling and noise regularization. Normal databases should match the study's imaging system, reconstruction software, radiopharmaceutical, and be representative of the patient population. ${ }^{4,9}$

ASNC has provided guidance for the optimal performance of nuclear cardiology with SPECT. ${ }^{10,11} \mathrm{~A}$ recent ASNC information statement gives recommendations on technology and imaging protocols that should be considered standard-of-care today. ${ }^{12}$ These recommendations include the adoption of low-dose imaging protocols and the use of high-sensitivity SPECT systems that can be used to achieve quality images with low radiation dose. As nuclear cardiology imaging laboratories strive to reduce the radiation burden to the patient, we must continually evaluate our procedures to make sure that we maintain a high standard of quality, produce high-quality images, and do not sacrifice diagnostic accuracy.

\section{Disclosure}

The authors have no conflict of interest.

\section{References}

1. Knuuti J, Bengel F, Bax JJ, et al. Risks and benefits of cardiac imaging: An analysis of risks related to imaging for coronary artery disease. Eur Heart J 2014;35:633-8.

2. Einstein AJ, Pascual TNB, Marcuri M, et al. Current worldwide nuclear cardiology practices and radiation exposure: Results from the 65 country IAEA Nuclear Cardiology Protocols Cross-Sectional Study (INCAPS). Eur Heart J 2015;36:1689-96.

3. Dorbala S, Blankstein R, Skali H, et al. Approaches to reducing radiation dose from radionuclide myocardial perfusion imaging. $\mathrm{J}$ Nucl Med 2015;56(592):599.

4. Rubeaux M, Xu Y, Germano G, et al. Normal databases for the relative quantification of myocardial perfusion. Curr Cardiovasc Imaging Rep 2016;9(8):22.

5. Einstein AJ, Blankstein R, Andrews $\mathrm{H}$, et al. Comparison on image quality, myocardial perfusion, and left ventricular function between standard imaging and single-injection ultra-low-dose imaging using a high-efficiency SPECT camera: The MILLISIEVERT study. J Nucl Med 2014;55:1430-7.

6. Nakazato R, Berman DS, Hayes SW, et al. Myocardial perfusion imaging with solid-state camera: Simulation of a very low dose imaging protocol. J Nucl Med 2013;54:373-9.

7. Van Dijk JD, Borren NM, Mouden M, et al. Effect of patientspecific minimum activity in stress myocardial perfusion imaging using CZT-SPECT: Prognostic value, radiation dose, and scan outcome. J Nucl Cardiol 2018;25:26-35.

8. Wetzl M, Sanders J, Kuwert T, et al. Effect of reduced photon count levels and choice of normal data on semi-automated image assessment in cardiac SPECT. Current issue.

9. Galt JR. New techniques, distinctive population, unique normal databases. J Nucl Cardiol 2018;25:1338-41.

10. Dorbala S, Ananthasubramaniam K, Chareonthaitawee P, DePuey EG, Eisntein AJ, et al. Single Photon Emission Computed Tomography (SPECT) myocardial perfusion imaging guidelines: 
Instrumentation, acquisition, processing, and interpretation. J Nucl Cardiol 2018. https://doi.org/10.1007/s12350-018-1283-y.

11. Henzlova MJ, Duvall WL, Einstein AJ, Travin MI, Verberne HJ. ASNC imaging guidelines for SPECT nuclear cardiology procedures: Stress, protocols, and tracers. J Nucl Cardiol 2016;23:60639. https://doi.org/10.1007/s12350-015-0387-x.
12. Abbott BG, Case JA, Dorbala S, Einstein AJ, Galt JR, Pagnanelli R, Bullock-Palmer RP, Soman P, Wells RG. Contemporary cardiac SPECT imaging-innovations and best practices: An information statement from the american society of nuclear cardiology. J Nucl Cardiol 2018;25:1847-60. 